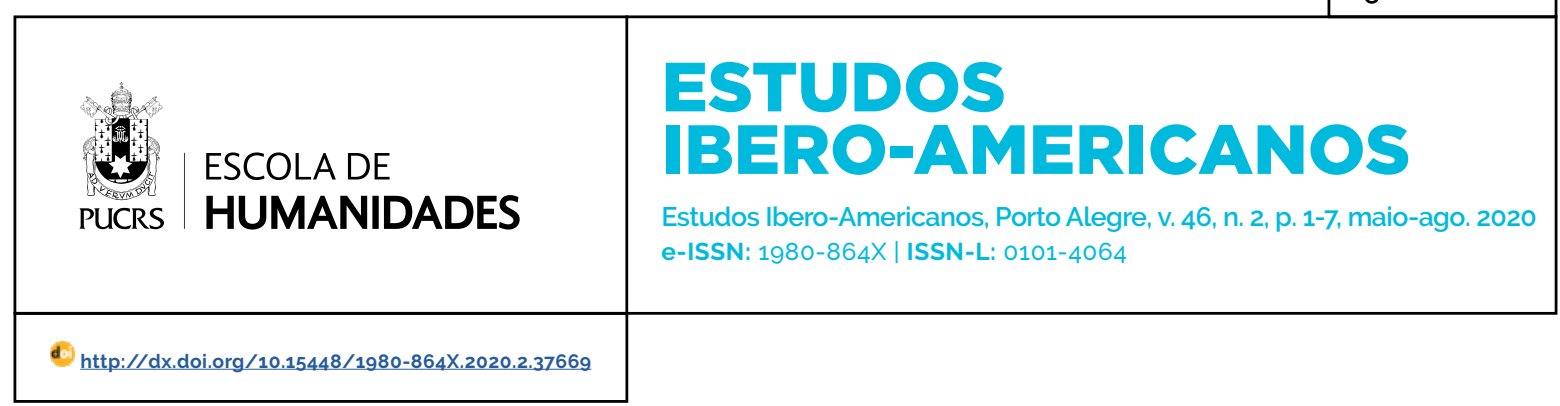

DOSSIÊ IMPRENSA, CULTURA E CIRCULAÇÃO DE IDEIAS

ENTREVISTA

\title{
História e Imprensa na América Latina: o processo de institucionalização de um campo de estudos - entrevista com Celia del Palacio Montiel
}

Historia y prensa en América Latina: el proceso de institucionalización de un campo de estudio - entrevista con Celia del Palacio Montiel

History and Press in Latin America: the process of institutionalizing a field of study interview with Celia del Palacio Montiel

\section{Marlise Regina Meyrer ${ }^{1}$ orcid.org/0000-0002-6446-7799 marlise.meyrer@pucrs.br}

\section{Helder V. Gordim da \\ Silveira ${ }^{1}$}

orcid.org/0000-0002-3262-1149 helders@pucrs.br

Recebido em: 13 abr. 2020. Aprovado em: 13 abr. 2020 Publicado em: 25 ago. 2020

\section{(c) (1)}

Artigo está licenciado sob forma de uma licença Creative Commons Atribuicão 4.0 Internacional.
Celia Del Palacio Montiel é investigadora e docente do Centro de Estudios de la Cultura y la Comunicación da Universidad Veracruzana. Dra. em Historia pela Universidad Autónoma de México. Membro do Sistema Nacional de Investigadores, nível III. Membro da Academia Mexicana de la Ciencia. Presidente e fundadora da Red de Historiadores de la Prensa en Iberoamérica (1999-2011). Membro do PEN Club Internacional Sección México e membro fundador da Academia Jalisciense de Ciencia. Desde 2018 é coordenadora do Grupo Temático Historia de la Comunicación, da Asociación Latinoamericana de Investigadores de la Comunicación ( $A L A / C)$. Sua especialidade é historia da imprensa regional do México, dirigiu o projeto "Violencia y medios de comunicación en Veracruz" com financiamento do Consejo Nacional de Ciencia y Tecnología. O produto mais recente desse projeto é o livro: Callar o Morir en Veracruz. Violencia y médios de comunicación durante el sexenio de Javier Duarte: 2010-2016, publicado en 2018. É autora de nove livros, coordenadora e coautora de outros dezesseis. Publicou, ainda, um grande número de artigos acadêmicos em revistas indexadas e de divulgação. Também é autora de quatro novelas históricas e um livro de relatos. Seus livros mais recentes são: Pasado y presente. 220 años de periodismo en Veracruz. (2015) e Callar o Morir en Veracruz. Violencia y medios de comunicación en el sexenio de Javier Duarte:2010-2016. (2018).

Marlise R. Meyrer: Desde finales de la década de 1960, vemos la proliferación y consolidación de los temas de la actualidad en la historiografia. Aproximadamente al mismo tiempo, fuimos testigos de la institucionalización de los estudios que tienen a la prensa como 
fuente y objeto de investigación. ¿Podría hablar un poco sobre la relación entre la Historia del Tiempo Presente y el creciente interés en el periodismo como objeto de estudio? Pensando especificamente en el contexto de la violencia en México a principios del siglo XXI y la creación de instituciones y grupos de investigación que son causa y consecuencia del aumento de publicaciones sobre el tema.

Celia del Palacio Montiel : En el caso de México, influyó mucho más la historia cultural y en particular la historia de los impresos, que permitió abordar el estudio sistemático de la prensa, que hasta antes de los años 80 , solo era tomada como fuente de la investigación histórica. Los historiadores más jóvenes comenzaron a interesarse en la producción, circulación y consumo de impresos, entre ellos, los periódicos. Por otro lado, los estudios de la comunicación, campo que estaba en sus inicios por aquellos años, también entendieron que era preciso indagar en torno a la producción, circulación y consumo de los productos mediáticos más allá la actualidad de los mismos. Entendieron que era preciso abordarlos desde una dimensión histórica. Muchos de los estudios históricos sobre radio, televisión y prensa fueron hechos por comunicólogos.

Creo que a finales de los años 90, creció el interés por consolidar este subcampo, a través de redes y grupos de trabajo en las asociaciones de historiadores y comunicólogos. Sin duda esto hizo crecer el número hasta entonces muy reducido de publicaciones y tesis sobre el tema.

Yo no creo que el interés sobre la prensa actual se derive de aquellos esfuerzos directamente ni que haya una relación con la historia del tiempo presente, que ha tenido relativamente pocos cultivadores en México. El creciente número de publicaciones sobre estudios del periodismo viene de otras matrices teóricas que también tuvieron origen y desarrollo en las décadas de los 80-90, desde los estudios de comunicación. Actualmente el interés por las condiciones de trabajo y riesgos de los periodistas ha crecido sustancialmente ante la violencia ejercida contra ellos, en un esfuerzo de entender las causas de dicha violencia y la búsqueda de posibles soluciones.

Marlise Regina Meyrer - Se puede decir que los estudios de la prensa todavía son cuestionados en cuanto a su afirmación como tema específico de estudios. ¿Usted considera que los estudios que utilizan la prensa como fuente y objeto de investigación en México hoy constituyen un campo disciplinario específico? ¿Qué factores / acciones corroboran en el proceso de consolidación de este campo de estudio?

Celia del Palacio Montiel : En México, la historia de los impresos y en particular la historia de la prensa se ha ido consolidando en los años recientes, a través de varias redes, como la que yo coordiné desde 1999: Red de Historiadores de la Prensa en Iberoamérica, que sigue celebrando sus congresos que convocan a decenas de investigadores. En otros países latinoamericanos, se han creado otras redes, como la Red de Historia de los Medios, en Argentina, o como la Red de Historiógrafos de la Prensa. Dentro de las asociaciones nacionales (AMIC) e internacionales (ALAIC) de la comunicación se han creado -y prevalecido- grupos de trabajo sumamente exitosos, tanto sobre Historia de la Comunicación, como de Estudios de Periodismo. La plataforma del Sistema Nacional de Investigadores en México, cuando pregunta por la subespecialización del historiador, da la opción "historia de la prensa". Esto me parece sumamente importante y lo considero como un avance en la consolidación del campo.

Marlise Regina Meyrer - Su proyecto Historia Comparativa de la Prensa Regional en México (1795 - 1950) fue pionero en los estudios de prensa en México desde una perspectiva regional y descentralizada, criticando la forma homogeneizadora de estudios de historiografía sobre el tema. En el libro: Siete regiones de la prensa en México (1792-1950), usted utiliza el concepto de "Región Periodística". ¿Podrias aclararnos este concepto? 
Celia del Palacio Montiel : Sigo a Eric Van Young cuando afirma que la región es "un espacio geográfico más grande que una localidad, pero más pequeño que un estado-nación, con una frontera para delimitarlo. Esa frontera depende del alcance de un sistema cuyas partes interactúan unas otras, más que con los sistemas externos" (Mexico's regions. Comparative history and development, Universidty of California, 1992, p. 3). Por ello me atreví a plantear el concepto "región periodistica", que funcionaba mejor para el siglo XIX, cuando las fronteras subnacionales actuales no existian como las conocemos hoy. Pero incluso en la época actual podría proponerse este concepto dentro de las entidades federativas muy atomizadas, como el estado de Veracruz, donde las ciudades medias están muy lejanas unas de otras, más cercanas a núcleos urbanos de otros estados. Estas regiones comparten hábitos de consumo, intereses particulares, han logrado consolidar sus propios mercados mediáticos $\mathrm{y}$, hasta cierto punto, una cultura periodistica compartida. A esto llamo una "región periodistica", en la cual las características de los medios guardan mayor similitud entre ellos, que con los de los medios de otras ciudades, estados, regiones. Esto a pesar del uso de nuevas tecnologías, redes sociales, adopción de modelos empresariales transnacionales, que pretenderian uniformar rutinas, agendas, formatos, etc. Algo en lo que insisto es que no debe asumirse que un estado con sus fronteras establecidas de manera un tanto arbitraria es lo mismo que una región periodistica. De cualquier forma, entiendo que es un concepto que requiere reformulación y una mayor justificación teórica y empírica.

Marlise Regina Meyrer - En su libro: Violencia y Periodismo Regional en México, once artículos analizan cómo se representa la violencia en la prensa. En el trabajo, su artículo sobre la región de Veracruz, al abordar las diferentes formas de violencia en la prensa regional, afirma que esta es una forma estructural y duradera de sumisión de los medios al gobierno, de clientelismo histórico con respecto a relaciones de los periodistas con el poder. En este sentido, ¿sería esta la misma perspectiva a largo plazo apropiada para los estudios sobre la violencia ejercida en la prensa "democrática" en toda América Latina?

Celia del Palacio Montiel : Creo que la prensa en cada pais debe analizarse tomando en cuenta las múltiples mediaciones que atraviesan su producción, circulación y consumo. Puedo afirmar que el clientelismo como resultado de la sumisión histórica de la prensa a un régimen autoritario como fue el priista durante casi 80 años en México, se naturalizó y corrompió las prácticas periodisticas hasta la médula. Esta situación se prolongó a nivel subnacional, en estados que conservaron el autoritarismo priista, como fue el régimen de Javier Duarte en Veracruz hasta 2016. A nivel nacional, después del año 2000, con la transición democrática, las prácticas comenzaron a cambiar y los teóricos como Manuel Guerrero y Mireya Márquez hablan de un "periodismo liberal-capturado": liberal en sus prácticas, en sus intenciones, en sus formatos, pero capturado por el estado, a través de convenios multimillonarios. En México el modelo "watch-dog" para la prensa nunca funcionó. Habría que analizar las caracteristicas históricas, políticas y sociales de cada pais y emprender estudios comparativos, que hacen mucha falta.

Helder V. Gordim da Silveira - Uno de los campos de su investigación se refiere a las condiciones de trabajo de los periodistas en las regiones de Veracruz, cuya precariedad usted señala como un factor de corrupción en el sistema, especialmente en lo que respecta al tratamiento de la violencia criminal. ¿Usted podría detallar esta relación? ¿Esta relación también sucede en otras regiones del pais y se convierte en objeto de investigaciones especificas en México?

Celia del Palacio Montiel : En la mayor parte de las regiones de México, los periodistas viven y trabajan en condiciones de grave precariedad y riesgo. Son muy vulnerables a la cooptación por parte, tanto de los gobiernos, como del 
crimen organizado. Esto es particularmente cierto en las poblaciones pequeñas, donde todo el mundo se conoce y la vigilancia de los actores armados (llámese autoridades o crimen y los grupos resultantes de la colusión entre ellos) es constante. Para estos periodistas más precarios, aunque no solamente a ellos, aplica más que nunca el "plata o plomo". En situaciones como esa, las opciones son limitadas: aceptar el dinero y pasar a formar parte de la nómina del grupo criminal, huir del lugar, abandonar el ejercicio periodístico/dejar de cubrir ciertos temas sensibles, o exponerse a la muerte violenta. Se han hecho múltiples estudios de un tiempo a esta parte sobre el impacto de la violencia sobre el ejercicio periodístico en diversos lugares de México, algunos de los cuales están contenidos en el libro que aqui se cita, pero en los últimos cinco años, la producción de artículos, libros, tesis de posgrado, sobre el tema desde distintos enfoques teóricos y metodológicos, ha crecido mucho. Sin embargo, aún hay "agujeros negros", regiones de las que se sabe poco y se publica menos: Guerrero, Oaxaca, la frontera sur, por ejemplo. También es importante mencionar el creciente interés de los propios periodistas y organizaciones nacionales e internacionales de defensa de los periodistas, de analizar y producir conocimiento desde su propia experiencia. No debe menospreciarse este esfuerzo.

Helder V. Gordim da Silveira - En su trabajo, la tensión universal contemporánea entre el carácter empresarial privado de las organizaciones periodísticas y la posición institucional de la prensa como un "cuarto poder" en un estado de derecho se percibe de manera concreta. ¿Podría contestarme si la violencia contra los periodistas proviene principalmente de factores relacionados con el polo privado de esta tensión, contrario a lo que generalmente se supone?

Celia del Palacio Montiel : Como he dicho más arriba, en México la prensa muy pocas veces ha podido ser un "cuarto poder": ha estado capturada económicamente por los regímenes políticos autoritarios. ¿De donde viene la violencia contra los periodistas? Es dificil de precisar en esta "niebla de la guerra económica" como la ha llamado Andreas Schedler, en la que se confunden los actores. El crimen organizado opera gracias a la connivencia o por lo menos permisividad e indolencia de ciertas autoridades, lo cual se traduce en impunidad. Los números que reportan las organizaciones internacionales de defensa a periodistas so muy claros: un enorme número de agresiones proviene de las autoridades-polícia, ejército, marina, etc. Por otro lado, es pertinente aclarar que los periodistas sufren de múltiples violencias, como lo he dicho en los trabajos recientes. Antes de la violencia directa, los periodistas son víctimas de la violencia estructural, ejercida por las propias empresas periodisticas privadas. Me refiero a los bajos salarios, inexistencia de programas de seguridad social, inseguridad laboral, despidos injustificados, no dotarlos de instrumentos ni recursos para realizar su trabajo, entre otros muchos. Los casos más graves provienen de empresas locales que no les dan salario, solo la "licencia" para exigir dinero de los políticos regionales a cambio de cobertura favorable. El gobierno, los gobiernos, contribuyeron a esta violencia al entregar grandes cantidades de dinero a los empresarios de los medios (cuando tuvieron los recursos) a cambio del mismo trato privilegiado, contribuyeron al no dotar de instrumentos claros a los mecanismos (pocos) de defensa de los periodistas y al no dotar de presupuesto suficiente a las instancias judiciales que podrian defenderlos. Ahora, ha contribuido al recortar aún más los presupuestos, al ejercer violencia simbólica constante contra los periodistas y defensores de derechos humanos críticos, al no proteger de ninguna manera a los cientos de trabajadores de los medios que han cerrado por falta de convenios publicitarios. ¿Quién agrede a los periodistas? Todos estos actores, públicos y privados. Todos sin excepción.

Helder V. Gordim da Silveira - Es bien sabido que el narcotráfico establece formas de dominación violenta y cooptación de estratos 
populares en el área cercana, estableciendo lo que generalmente se llama un "estado paralelo" en las regiones metropolitanas. ¿Tendrian las políticas públicas de censura legítima tratadas en su trabajo como uno de sus objetivos la lucha contra esta relación entre el narcotráfico y los estratos populares de las periferias urbanas? ¿Cómo usted evalúa este tema?

Celia del Palacio Montiel : No, no lo creo. Si entendo bien su pregunta, en estados como Veracruz y otros donde existió un régimen autoritário subnacional, la censura iba dirigida a conservar una ilusión de calma, de gobernabilidad, como lo dijo más de una vez el gobernador de Veracruz: aqui no passa nada. Por supuesto en dicha censura hay también un componente de censura de los propios grupos del crimen organizado, muchas veces en connivencia con las fuerzas de seguridad. Hay evidencia en muchos lugares de Veracruz, en muchos lugares de México, de que en las salas de redacción hubo un "gatekeeper" del grupo criminal dominante en la zona, que ordenó directa o indirectamente lo que podía o no podía publicarse.

Helder V. Gordim da Silveira - En algunos trabajos usted se refiere a las redes sociales como posibles fuentes alternativas de información dadas las diversas formas de censura y corrupción en la prensa tradicional para abordar la violencia. Sin embargo, ¿No serian, en el caso del narcotráfico, las redes virtuales un espacio para la interacción social aún más vulnerable que la prensa actual del poder privado? Por supuesto, ¿Las redes no constituyen, por ejemplo, un territorio privilegiado para el glamour social del crimen?

Celia del Palacio Montiel : Creo que es necesario tomar en cuenta el contexto. Tal vez es complicado entender, fuera de Veracruz y algunos otros estados en México donde hubo una total opacidad sobre lo que ocurría, la importancia de las redes sociales. En otras partes de México, como Guadalajara, la Ciudad de México, los periódicos sensacionalistas rebosaban sangre y sórdidos detalles sobre los crimenes del día. Como consigno en varios trabajos, lo que ocurrió en Veracruz fue lo opuesto. La académica Guadalupe H. Mar lo llamó un paso "de la nota roja a la nota rosa". En los periódicos veracruzanos ya no se reportaba lo que estaba ocurriendo, sino versiones edulcoradas de la violencia. Lo más escandaloso era un accidente de tránsito. Eso no daba ninguna tranquilidad, ya que la gente SABÍA por experiencia personal que en su barrio, en su colonia, había ocurrido una balacera, una persecución con varios muertos, y nada de eso aparecía en los periódicos. Recientemente en charlas con familiares de desaparecidos en Veracruz, me confirmaban que la consulta en Facebook de páginas especializadas en crimenes, les era fundamental para intentar encontrar a sus familiares. Los periódicos son pocas veces las fuentes a las que acuden para saber lo que ocurre en su localidad. Detrás de algunas de esas páginas están grupos del crimen organizado, pero detrás de otras, están los periodistas que ahi publican todo lo que no se les permite publicitar en los medios tradicionales. Ahora bien, es cierto que las redes han servido para alimentar "el glamour" del crimen organizado, como usted lo llama, pero el crimen consideró más glamoroso y relativamente fácil, al menos en un época, colonizar los medios tradicionales y obligarlos a adoptar su agenda. El público de esos periódicos no eran los jóvenes pobres de la periferia: eran los políticos y sus contrincantes. Como apunta Dan Hallin, el prestigio de los periódicos en nuestros paises ha servido siempre a ese propósito: hablar a las elites, a los poderosos, sean gobierno o poderes fácticos.

Suscribo de alguna manera lo que los periodistas me dijeron: quienes hicieron el "Acuerdo contra la violencia" en un escritorio de la Ciudad de México, procurando que no se hiciera apología del crimen, no saben lo que ocurre en el terreno. La situación que se vive en las regiones más pobres del estado del Veracruz, en las periferias de muchas ciudades de México, es mucho más compleja y no se puede explicar en términos binarios. 
Marlise Regina Meyrer - Podemos decir que uno de los problemas en los estudios sobre la prensa en América Latina sigue siendo su débil discusión teórico-metodológica. Esta situación ha cambiado con el crecimiento de los trabajos académicos sobre el tema en los últimos años. Sin embargo, en casi todos estos trabajos, predomina un aparato metodológico teórico basado en autores europeos, con énfasis en Roger Chartier, Pierre Bourdieu, Robert Darton y Jonh Thompsom John Thompson. Si bien no se puede negar la importancia de estas contribuciones, ¿contemplan las especificidades regionales de América Latina? ¿Cómo promover una mayor producción y circulación del pensamiento latinoamericano en estudios de la prensa?

Celia del Palacio Montiel : Creo que además de los académicos que se citan, hay que considerar que existe una influencia importante de los estudios de la comunicación latinoamericanos en los estudios sobre prensa. Personalmente, en mis trabajos he citado repetidamente la contribución fundamental de Enrique Sánchez Ruiz con su enfoque "histórico-estructural" para abordar el estudio de la prensa, que merecería, en efecto, un mayor reconocimiento. En los últimos años, se ha aprovechado crecientemente el legado de Jesús Martin Barbero para abordar las mediaciones en el estudio de las audiencias. Desde la historia cultural, es importante la contribución de Marialva Barbosa y de Mirta Varela. Así mismo, los jóvenes mexicanos estudiosos del periodismo han contribuido grandemente con sus acercamientos al periodismo post-transición democrática, las rutinas periodisticas, las empresas mediáticas, legislación sobre medios,el abordaje con un enfoque de género el trabajo y riesgos en el periodismo o bien, a aplicar las matrices teóricas de la ciencia política a los estudios de la prensa subnacional actual. Pienso en María Elena Hernández, Salvador de León, Mireya Márquez, Frida Rodelo y Grisel Salazar, entre otros muchos.

Desde la historia, lamento decir que que los acercamientos latinoamericanos no han variado mucho y que se siguen haciendo estudios monográficos sobre un periódico en particular o un lugar y periodo particular, sin hacer las conexiones con el resto de América Latina o con el mundo. Creo que los análisis situados son fundamentales, pero a veces siento que estamos en el mismo sitio que en la década de 1990 , cuando cualquier contribución desde lo local era muy apreciada porque era prácticamente lo único que había. Falta una reflexión teórica y metodológica más profunda y (auto) crítica sobre el quehacer, retomando lo que ya se hizo y planteando nuevos horizontes.

Marlise Regina Meyrer - Actualmente, estamos presenciando la proliferación en los medios de comunicación de discursos de odio con prácticas de violencia contra periodistas, derechos humanos y una difusión generalizada de información falsa, muchas veces como una estrategia del propio gobierno. Como ejemplo, podemos citar a Donald Trump y Jair Bolsonaro, que eligieron a los medios de comunicación como enemigos del pueblo, en un movimiento para deslegitimar las noticias. ¿Cómo ve usted este escenario y cómo pueden los estudiosos de los medios de comunicación contribuir a la defensa de la libertad de prensa?

Celia del Palacio Montiel : Esto ocurre de la misma manera en México. El presidente Andrés Manuel López Obrador también ha catalogado a los periodistas como enemigos y los ha llamado "chayoteros" (vendidos), entre otros adjetivos denigrantes. El clima hacia los periodistas criticos (en particular hacia las mujeres periodistas, hay que decirlo) es muy hostil. De hecho, hay un clima muy hostil hacia cualquiera que se muestre critico al régimen, incluidos los académicos. Esto empeora, además, en un pais cada vez más polarizado donde quedan pocos espacios para el debate público.

A los estudiosos de los medios toca analizar a profundidad lo que ocurre, desde la academia, denunciar los ataques a la libertad de expresión y presentar la evidencia en los foros más allá del reducido espacio académico. Asi mismo, enseñar a los alumnos a distinguir la información falsa, a 
analizar criticamente la situación y desempeñar un papel como intelectuales públicos, que no siempre nos ha venido muy bien ni hemos querido desempeñar y que sin embargo resulta fundamental hoy.

Dirección:

Marlise Regina Meyrer

Pontificia Universidade Católica do Rio Grande do Sul

Av. Ipiranga, 6681

Partenon 90619-900

Porto Alegre, RS, Brasil 